\title{
Wetting and evaporation of a sessile drop under an external electrical field: A review
}

\author{
V. Vancauwenberghe ${ }^{\mathrm{a}, *}$, P. Di Marco ${ }^{\mathrm{b}}$, D. Brutin ${ }^{\mathrm{a}}$ \\ a Aix-Marseille University, IUSTI UMR 7343 CNRS, 13453 Marseille, France \\ ${ }^{\mathrm{b}}$ LoThAR, Department of Energy and Systems Engineering, University of Pisa, Pisa, Italy
}

\section{G R A P H I C A L A B S T R A C T}

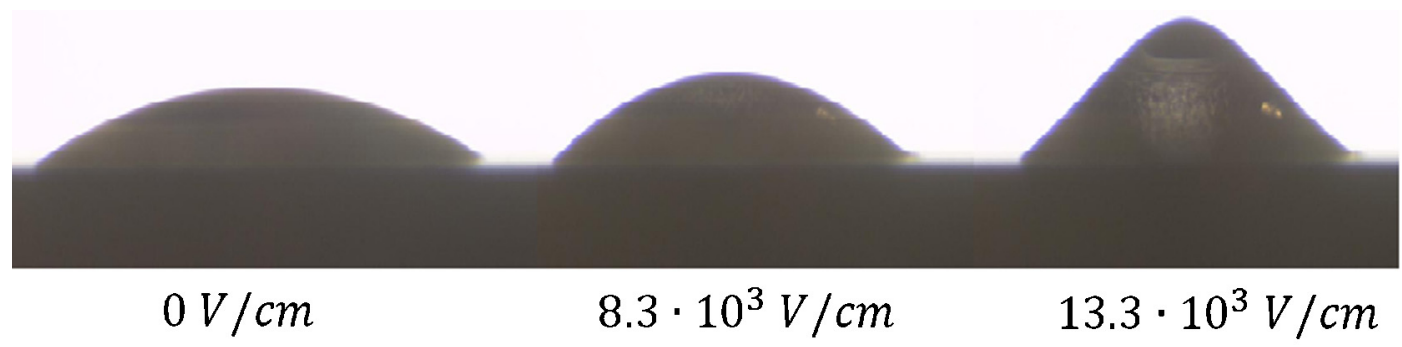

\section{H I G H L I G H T S}

- Electrical field modify sessile drop interfaces

- Wetting and evaporation are influenced by few $\mathrm{kV} / \mathrm{cm}$ fields.

- Evaporation enhancement is potentially important if wetting is controlled.

\section{A R T I C L E I N F O}

\section{Article history:}

Received 7 April 2013

Received in revised form 29 April 2013

Accepted 30 April 2013

Available online 9 May 2013

\section{Keywords:}

Evaporation

Electric field

Contact angle

Sessile drop

\begin{abstract}
A B S T R A C T
The enhancement of heat and mass transfer using a static electric field is an interesting process for industrial applications, due to its low energy consumption and potentially high level of evaporation rate enhancement. However, to date, this phenomenon is still not understood in the context of the evaporation of sessile drops. We synthesise the current research concerning the effect of an electric field on sessile drops with a focus on the change of contact angle and shape and the influence of the evaporation rate. We also discuss the future prospects of this research field.
\end{abstract}

(c) 2013 Elsevier B.V. All rights reserved.

\footnotetext{
* Corresponding author. Tel.: +33491106986.

E-mail addresses: valerie.vancauwenberghe@polytech.univ-mrs.fr, vavcauwe@ulb.ac.be (V. Vancauwenberghe), p.dimarco@ing.unipi.it (P. Di Marco), david.brutin@univ-amu.fr (D. Brutin).
}

\section{Introduction}

Over the past few decades, the evaporation of sessile drops has been the subject of a great deal of interest, due to its increasing use in heat and mass transfer applications and industrial processes. Most of these investigations have been focused on the complexity of the physical dynamics, such as the influence of the substrate surface on the drop wettability, the evaporation flux at the interface and 
the triple line, and the influence of the substrate temperature on the evaporation rate [1-6].

Under normal gravity, different physical mechanisms drive the evaporation of small drops: heat transfer by convection, heat transfer induced by the substrate conduction, diffusion of the vapour around the drop in the gaseous phase, and the molecular interactions between the drop and the substrate that change the wettability of the drop. From the existing literature, it is generally assumed that drop evaporation is mainly controlled by the diffusion of molecules from the liquid-gas interface to the surrounding gas $[7,8]$. This quasi-steady mechanism is expressed by the Laplace equation. For small contact angle $\left(\theta<90^{\circ}\right)$, the evaporation flux $J$ is given by the Fick's law: $J=-D \nabla c$, where $D$ is the coefficient of diffusion and $c$ corresponds to the concentration $[9,10]$. However, the rate of mass loss has been expressed for an arbitrary contact angles by Popov [11]:

$-\frac{d m}{d t}=\pi R D\left(c_{s}-c_{\infty}\right) f(\theta)$

where $m$ is the mass of the drop, $R$ is the wetting radius, $t$ is the time, $c_{s}$ is the saturate concentration of vapour at the drop interface, $c_{\infty}$ is the concentration of vapour in the air and $f(\theta)$ is a function of the contact angle.

The effect of a static electric field is of great interest in many applications, including mass and heat transfer [12,13], particle manipulation [14], coating [15] and drying [16]. Indeed, the generation of an electric field is a cost-effective solution due to its low power consumption.

The enhancement of the heat transfer in the evaporation process by the application of an electric field can be useful on earth to improve the evaporation rate, as well as in space, under weightlessness, to replace the absence of the convective effect. Thus, the objective of research in this area is to overcome the diffusion limitation on the evaporation rate by applying an external electric field. Moreover, in the scope of the nanoparticle deposition by evaporation, which is limited on earth due to the presence of capillary convection and gravity (the coffee ring effect), the use of an electric field would provide a tool to control the pattern formation. The applications are various on earth, as well as in space, regarding the possibilities of heat transfer enhancement, such as cooling using drop evaporation in space or the uniform deposition of nanoparticles that is required for almost all industrial applications (e.g., coating, printing, DNA mapping).

When a drop is exposed to an electric field, its equilibrium shape is established by the balance between the surface tension and the external force corresponding to gravity and the electric field. In the case of a sessile drop, the surface tension tends to make the drop spherical, whereas gravity tends to flatten it. Typically, the external field tends to elongate the drop along the field direction. The augmented Young-Laplace equation describes the mechanical equilibrium between the surface tension, the electric field, and gravity [17-19]

$\gamma\left(\frac{1}{R_{1}}-\frac{1}{R_{2}}\right)=\Delta P_{0}+(\Delta \rho) g z+\Delta P_{e}$

where $\gamma$ is the surface tension, $R_{1}$ and $R_{2}$ are the principal radii of curvature, $\Delta P_{0}$ corresponds to the difference of pressure across the drop interface, $\Delta \rho$ is the density difference across the interface, $g$ is the gravitational acceleration, $z$ is the vertical distance from the reference and $\Delta P_{e}$ is the electrical pressure corresponding to the difference in the normal component of the Maxwell stress tensor across the interface.

The presence of an electric field affects the wettability of liquids according to Eq. (2). The change of the contact angle, called the electrowetting effect, is caused by a potential applied to the interface of the three-phase system $\varphi_{s l}$. This phenomenon can be

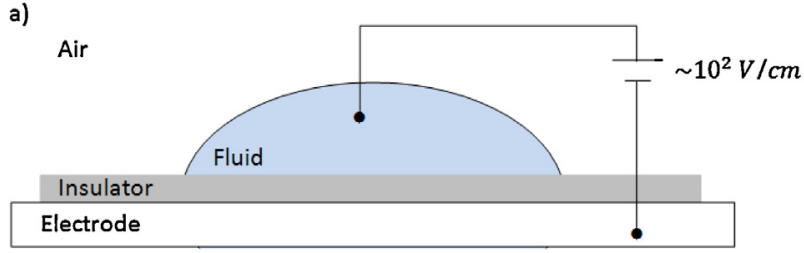

b)

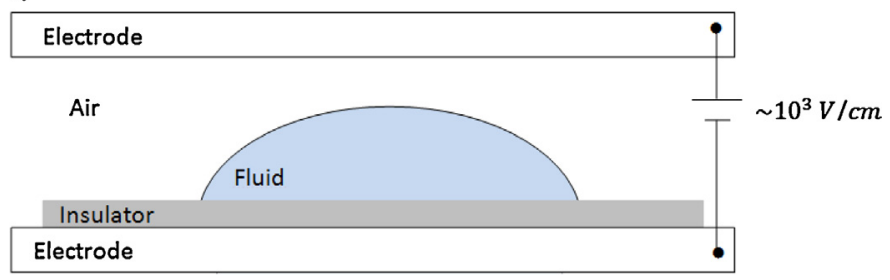

Fig. 1. Schematic of the electrode configuration. (a) The electric field is established inside the drop. The upper electrode is in contact with the drop volume. (b) The electric field is established outside the drop.

derived from the Lippmann equation using the approximation of a parallel capacitor for the solid-liquid interface and assuming the differential capacitance of the interface $C_{s l}=\epsilon_{0} \epsilon / d(\epsilon$ corresponding to the electric permittivity of the dielectric layer beneath the liquid drop and $d$ corresponding to its thickness) $[15,20,21]$ :

$\cos \left(\theta_{e}\right)=\cos \left(\theta_{0}\right)+\frac{C_{s l}\left(\varphi_{s l}-\varphi_{s l}^{0}\right)^{2}}{2 \gamma_{s l}}$

Here, $\theta_{e}$ is the contact angle under the electric field, $\theta_{0}$ is the contact angle without the field, and $\gamma_{s l}$ is the interfacial tension of the solid-liquid phase at the zero potential $\varphi_{s l}^{0}$.

An electric field can be established in two ways, as shown in Fig. 1. In the first case, the electric field is directly created inside the liquid drops. One electrode is placed in contact with the drop surface. The applied potential between the two electrodes is several hundred volts. Many theoretical, experimental, and numerical studies based on this configuration have been performed for different applications $[15,22,23]$ such as in microfluidics (actuation and drop manipulation) [24-28], optics [29,30], microelectromechanics [31-33] and electrical engineering [34-37]. However, because the electrodes must be in contact with the liquid, such applications require a precise adjustment of the experimental setup and may not be convenient for large processes or those involving volume changes.

In the other case, the electric field can be established between two plate electrodes without contact with the drop. This configuration is less popular than the first due to the order of magnitude of the applied potential, which is in the range of several $\mathrm{kV}$ (electric field $\sim 1-10 \mathrm{kV} / \mathrm{cm}$ ) and it constitutes a small part of the literature. However, this setup may be the most appropriate for evaporation applications.

This review encompasses the existing literature about the effect of an electrical field established by two plate electrodes on sessile drops. First, the investigations about the influence of the electric field on the contact angle and the drop shape are discussed. Next, the studies about the enhancement of evaporation under an electrical field are summarised. Finally, the review is concluded with a discussion of possible future investigations.

\section{Changes induced by an electric field}

When a dielectric fluid is placed in an uniform electric field $E$, a volumic electric force acts thereon. It is expressed by Eq. (4) [38]

$f_{e}=\rho_{e} E-\frac{\varepsilon_{0}}{2} E^{2} \nabla \varepsilon_{r}+\frac{\varepsilon_{0}}{2} \nabla\left(E^{2} \frac{d \varepsilon_{r}}{d \rho} \rho\right)$ 


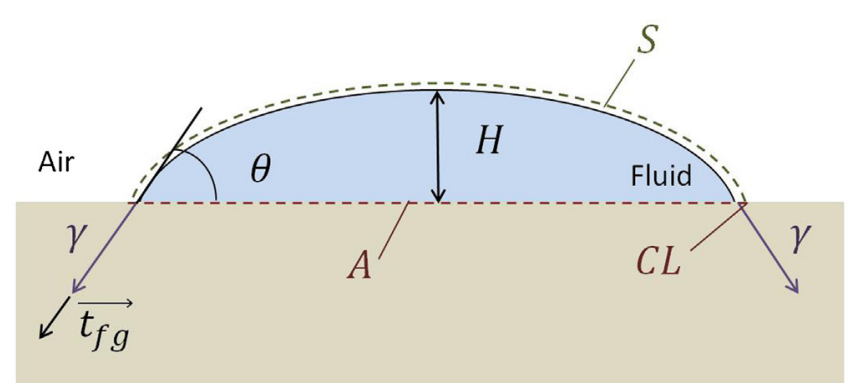

Fig. 2. Schema of the drop

The first term of this sum corresponds to the Colomb's force that is connected to the free charge density of the fluid $\rho_{e}$. This force generally predominates over the other forces in presence of free charge in the fluid. The second terms represents the dielectrophoretic force that results from the non-uniformity of the relative dielectric permittivity $\varepsilon_{r}$ under the electric field. The last term corresponds to the electrostriction force that acts on the fluid when electric field is non-uniform. The dielectrophoretic and electrostriction are independent of the field polarity [39].

The volumic electric force in and around a fluid interface can be reformulated as the divergence of the Maxwell stress tensor $\overline{\overline{T_{e}}}$ $[38,40,41]$

$f_{e}=\operatorname{div} \overline{\overline{T_{e}}}$

where its components are

$t_{i k}=\varepsilon_{0} \varepsilon_{r} E_{i} E_{k}-\frac{1}{2} \varepsilon_{0} E^{2}\left[\varepsilon_{r}-\frac{d \varepsilon_{r}}{d \rho} \rho\right] \delta_{i k}$

Thus the resulting electric force $F_{e}$ acting on the surface $S$ of a drop is given by Eq. (7). This force can be evaluated when the the electric field at the drop interface is known [46].

$F_{e}=\iint_{S} \overline{\overline{T_{e}}} \cdot n d S$

where $n$ is the normal outward unit vector.

When a drop sitting on a flat surface (see Fig. 2) is subjected to an electric field, the equilibrium can be calculated, for a stationary case, by considering the momentum balance given by Eq. (8).

$$
\begin{gathered}
\int_{C L} \gamma_{g l} \overrightarrow{t_{g l}} d L+\int_{V} \rho_{l} \vec{g} d V+\int_{S} \overline{\overline{T_{e, g}}} \cdot \vec{n} d S+\int_{A} \overline{\overline{T_{e, l}}} \cdot \vec{n} d A \\
-\int_{S} p_{g} \cdot \vec{n} d S-\int_{A} p_{l} \cdot \vec{n} d A=0
\end{gathered}
$$

Where $A$ is the drop base area, $C L$ is the contour of the drop base, $S$ is the area of the gas-liquid interface, $V$ is the drop volume and the subscripts $l$ and $g$ refer to the liquid drop and the surrounded gas, considered here to be air. The integrals represent respectively the adhesion force, the drop weight, the electric force at the gas-liquid interface, the electric force at the drop base and the force caused by the pressure at the gas-liquid interface $p_{g}$ and the base of the drop $p_{l}$.

The momentum balance can be simplified by considering that the value of the electric stress tensor is zero at the droplet base, which is grounded:

$\frac{\pi D^{2}}{4}\left(p_{f}-\rho_{g} g H\right)=V\left(\rho_{l}-\rho_{g}\right) g+\pi D \gamma \sin (\theta)+\int_{S} \overline{\overline{T_{e, g}}} \cdot \vec{n} d S$

where $D$ is the drop base diameter, $H$ is the drop height and $V$ is the drop volume.
Eq. (9) corresponds to

$F_{p}=F_{w}+F_{\gamma}+F_{e}$

where the corresponding forces are respectively the internal overpressure, the weight, the adhesion and the electric force [46].

The influence of an electric field on the contact angle and wetting radius of a drop must be known to be able to predict the evaporation rate given by Eq. (1). This section collects the studies based on the action of an external electric field (such the configuration in Fig 1b) with regard to three factors: the contact angle, shape, and evaporation rate.

\subsection{Influence of an electrical field on the wetting angle of a drop}

Only a few authors have studied the effect on the contact angle and the interface when an electric field is established outside a liquid drop (shown in Fig. 1b). As depicted in Eq. (1), the evaporation rate of a sessile drop is a function of $\theta$, which changes under an electric field. To predict this angle change, the Lippmann equation is proposed and has been fully implemented by many experimenters, who have correlated empirical results with a small variation in the contact angle, especially in electrowetting, as represented in Fig. 1a) $[15,22,23,42,43]$. In fact, the Lippmann equation considers the effect of the electric field on the drop wettability as a consequence of interface polarisation such as the classical capillary effect. This relationship supposes that the surface tension of the solidvapour and liquid-vapour phases, $\gamma_{s v}$ and $\gamma_{l v}$, are not affected by the electric field. This expression may not be considered for systems with a dielectric multilayer, nonaqueous liquids, and different electrolyte concentrations [44]. Digilov et al. proposed that the change in the contact angle may be due to the reduction of the line tension caused by the charge redistribution along the contact line of the three phase $[20,45]$.

The experimental studies summarised in Table 1 , focused on the change in the contact angle for dielectric liquids under an external electric field varying from 1 to $25 \mathrm{kV} / \mathrm{cm}$. The overall mean of these results suggested an increase or decrease in the contact angle with increasing magnitude of the electric field [17,45-50].

In their works on the lift-off of a conducting drop, Roux et al. $[47,48]$ observed a decrease in the contact angle of a water drop placed on the lower plate electrode of a condenser surrounded by a mineral oil with a high relative permittivity. Their investigations focused on the development of a theoretical model predicting the electric field threshold. They highlighted the effect of the contact angle hysteresis that induces an elongation of the droplet under the electric field without any movement along the contact line, decreasing the contact angle.

Roero [50] studied the shape and contact angle of sessile water drops on various substrates subjected to an electric field to understand the generation of sound emission from wet highvoltage transmission lines. In his experiments, three substrates that received different treatments (untreated, hydrophobic, and hydrophilic) were used and the variation of sessile water drops of various volumes and their instabilities under an increasing electric field were measured. The contact angle decreased with increasing electric field. Fig. 3 illustrates his observation.

Bateni et al. [17,45,51,52] were interested in the change of the interfacial tension of a droplet under an external field. Based on experimental results (see Fig. 4), they developed a new methodology, called ADSA-EF (Axisymmetric Drop Shape Analysis - Electric Field), to study the drop shape and surface tension under an external electric field that will be expanded in Section 2.2. An increase in the surface tension of different conducting liquids with the increase in the electric field was observed, and the analysis of the regression suggested that this increase is proportional to the square root of the electric field strength. Moreover, they highlighted some 
Table 1

Summary of the effect of an electric field on the contact angle $\theta_{0}$ of liquid drops

\begin{tabular}{|c|c|c|c|c|c|c|}
\hline Author & Liquids & Substrate & $\begin{array}{l}\text { Maximal electric } \\
\text { field } 10^{3} \mathrm{~V} / \mathrm{cm}\end{array}$ & $\theta_{0}$ & $\begin{array}{l}\text { Electric field influence } \\
\text { on the contact angle }\end{array}$ & Reference \\
\hline Di Marco & Ethanol & PTFE & 13.3 & 35 & Increase & [46] \\
\hline Roux & $\begin{array}{l}\text { Water + Tween } \\
\mathrm{NaCl} \text { solution }\end{array}$ & $\begin{array}{l}\text { Hydrophobic substrate } \\
\text { CNT }\end{array}$ & $\begin{array}{l}1.4 \\
1.5\end{array}$ & $\begin{array}{l}160 \\
162\end{array}$ & $\begin{array}{l}\text { Decrease } \\
\text { Decrease }\end{array}$ & $\begin{array}{l}{[48]} \\
{[47]}\end{array}$ \\
\hline Roreo & $\begin{array}{l}\text { Deionized water } \\
\text { Deionized water }\end{array}$ & $\begin{array}{l}\text { Stainless steel } \\
\text { Hydrophobic substrate }\end{array}$ & $\begin{array}{l}15.0 \\
15.0\end{array}$ & $\begin{array}{l}72 \\
98\end{array}$ & $\begin{array}{l}\text { Decrease } \\
\text { Decrease }\end{array}$ & $\begin{array}{l}{[50]} \\
{[50]}\end{array}$ \\
\hline Bateni & $\begin{array}{l}\text { Deionized water } \\
\text { Distilled water } \\
\text { Formamide } \\
\text { Propylene-carbonate } \\
\text { Alcohols }\end{array}$ & $\begin{array}{l}\text { Hydrophilic substrate } \\
\text { Stainless steel } \\
\text { Stainless steel } \\
\text { Stainless steel } \\
\text { PTFE }\end{array}$ & $\begin{array}{r}25.0 \\
5.6 \\
5.6 \\
5.6 \\
8.0\end{array}$ & $57-75$ & $\begin{array}{l}\text { Decrease } \\
\text { Increase } \\
\text { Increase } \\
\text { Increase } \\
\text { Increase }\end{array}$ & $\begin{array}{l}{[50]} \\
{[17]} \\
{[17]} \\
{[17]} \\
{[45]}\end{array}$ \\
\hline
\end{tabular}

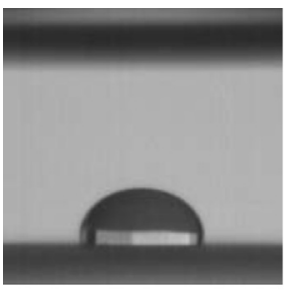

$0 \mathrm{~V} / \mathrm{cm}$

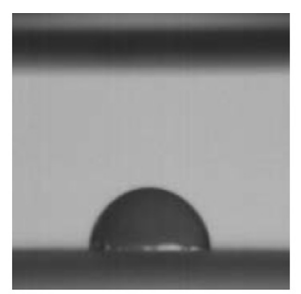

$8.5 \cdot 10^{3} \mathrm{~V} / \mathrm{cm}$

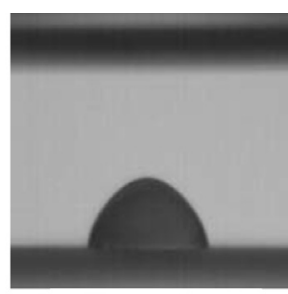

$10.0 \cdot 10^{3} \mathrm{~V} / \mathrm{cm}$
Fig. 3. Water droplet of $80 \mu \mathrm{l}$ deformed by the electric field. The substrate was an aluminium surface sandblasted and subjected to a hydrophobic treatment. The electrode spacing was $10 \mathrm{~mm}[50]$.

influencing factors, such as the liquid polarity and the size of the liquid molecules, and the lack of effect of the direction of the electric field. They also suggested that the observed increase in the contact angles is due to a bulk effect rather than an interfacial effect [45].

Further investigation is required to interpret the existing results, including the influence of the liquid and the substrate properties (conductivity, permittivity, wettability, surface treatment, etc.). Moreover, the effect of the electric field on the contact line has not been considered. However, the contact line can be pinned to the surface [17], or less. This may change contact angle evolution when the bubble is elongated by electric field and may explain differences in experiments.

\subsection{Influence of an electric field on drop shape}

The effect of an electrostatic field on the shape of a drop is a well-known phenomenon, elongating the drop along the direction of the field, as shown in Fig. 6. In 1882, Lord Rayleigh pioneered an investigation of the interaction of charges and electric fields on liquids drops. He theoretically derived the critical amount of charges required to destabilise an isolated, surface-charged, spherical drop [53]. His work comprised the beginning of electrohydrodynamics [54].

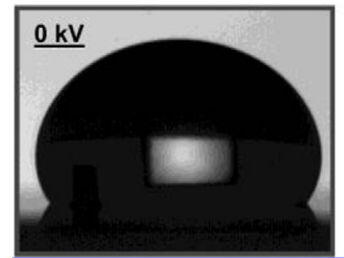

$0 \mathrm{~V} / \mathrm{cm}$

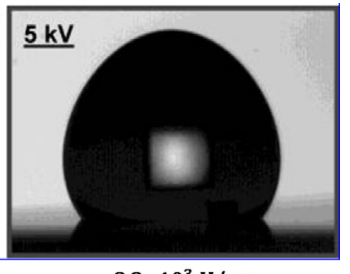

$8.3 \cdot 10^{3} \mathrm{~V} / \mathrm{cm}$
Fig. 4. Deformation of water drops under an electric field. The substrate was Teflon silicon wafers, and the electrode spacing was $6 \mathrm{~mm}$. The two drops did not have the same volume [51].
Over the past three decades, the shape and the stability of sessile drops under electric fields has been analysed in many works. Rosenkilde [55] examined the equilibrium and stability of an incompressible dielectric drop subjected to a uniform electric field. Assuming the drop to be ellipsoidal, he proposed a relationship between the equilibrium deformation, the strength of the electric field, the drop dimensions, and the surface tension. The cause of the instability for high fluid permittivities has also been presented [55].

Miksis [56] proposed a numerical model to compute the shape of an axisymmetric drop under a DC electric field, producing good agreement with experiments. His results show a critical value of the dielectric constant under which the drops elongated and retained their originally spheroidal shape. Above this critical value, the drop developed a conical shape.

In their numerical works, Basaran et al. $[41,57,58]$ calculated the shape and the stability of axisymmetric pendant and sessile drops or bubbles considering that the contact line is fixed or prescribed. Their first calculations addressed the assumption that conducting drops are linearly polarisable materials [41,57]. The equilibrium shape and stability of the drops are determined by simultaneously solving the Laplace and Young-Laplace equations. A study considering the non-linear polarisation of the drops was performed by substituting the Maxwell equation by the Laplace equation [58]. Their computations revealed the importance of varying the plate condenser spacing, which affects the electric field strength and the drop size.

Reznik et al. [59] distinguished three scenarios of droplet shape evolution under strong electric fields determined by the values of the electric bond number and based on the numerical solution of the Stokes equations. Under a weak electric field, the droplets are subjected to Maxwell stresses and exhibit steady-state shapes. Under strong electric fields and for low static contact angles, the droplets acquire a conical shape and jetting sets in from the droplet tip. The final scenario corresponds to the detachment of an almost whole droplet (i.e., a dripping phenomenon) that results from strong electric field and a contact angle exceeding a critical value. The numerical model was in good agreement with the experimental results for polymer droplets [59].

Bateni et al. [17,45,51,52] based their methodology ADSA-EF on an assumption of an axisymmetric drop shape. ADSA-EF can be used to measure the surface tension of a drop under an electric field with and without the gravity effect. From the Young-Laplace equation described by Eq. (2), they consider the electrical pressure $\Delta P_{e}$ which, depends on the permittivity of the fluids and the strength and the direction of the electric field, as following [51,52]

$\Delta P_{e}=\frac{1}{2}\left[\varepsilon_{0} E_{n}^{(g) 2}-\varepsilon_{0} \varepsilon_{r} E_{n}^{(l) 2}+\varepsilon_{0}\left(\varepsilon_{r}-1\right) E_{t}^{2}\right]$ 


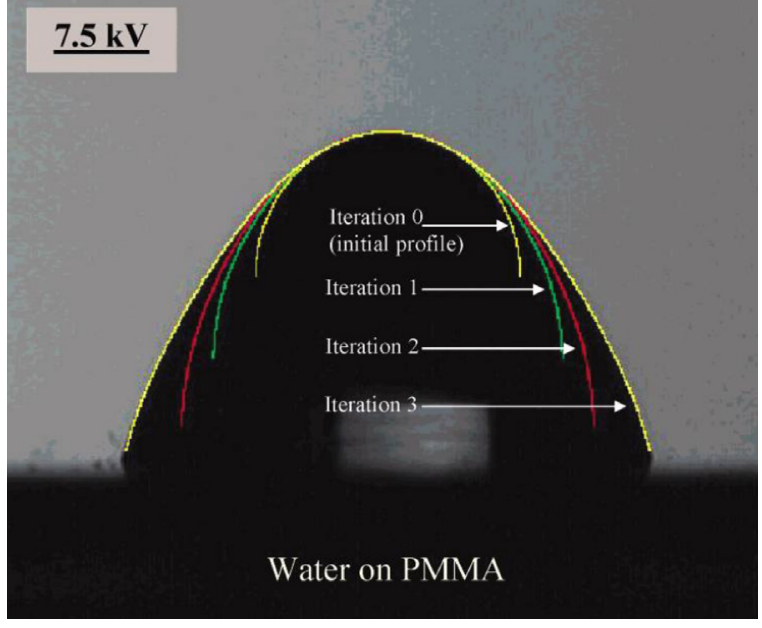

Fig. 5. Prediction of the drop shape by iterative calculations [51].

where $E_{t}$ and $E_{n}$ are respectively the tangential and the normal components of the electric field at the surface of the drop and the superscripts $l$ and $g$ refer to the drop liquid and the surrounded fluid seen as air. When the drop is a conducting fluid, there is no electric field inside the drop and the Young-Laplace equation is simplified as

$\gamma\left(\frac{1}{R_{1}}-\frac{1}{R_{2}}\right)=\Delta P_{0}+(\Delta \rho) g z+\frac{1}{2} \varepsilon_{0} E_{n}^{(g) 2}$

From Eq. (12) and the electrostatic field module, they numerically predicted the drop shape with an iterative scheme as shown in Fig. 5.

Di Marco et al. [46] evaluated the electric force acting on a sessile ethanol drop interface and compared the experimental results with a theoretical model based on the equilibrium action of a sessile drop, taking into account the electric field and gravity. The experimental setup is composed of a flat aluminium plate equipped with an orifice for droplet injection. The plate is electrically grounded and coated with a Teflon layer. A washer-shaped stainless-steel electrode, $4 \mathrm{~mm} \times 15 \mathrm{~mm}$, is laid parallel to the plate at a distance of $6 \mathrm{~mm}$ and connected to the positive pole of a high-voltage supply, which can provide up to $8 \mathrm{kV}$ DC. The resulting electric field in the cavity has an average strength of $13.3 \times 10^{3} \mathrm{~V} / \mathrm{cm}$.

The shape of the obtained droplets is shown in Fig. 6. The drops subjected to an electric field are elongated in the electric field direction due to the alteration of the interface curvature by the local electric stress, as reported previously. The contact angle and the curvature at the drop apex increase with increasing electric field strength [46].

The profile of the droplets and their geometric parameters (contact angle, drop dimensions, and volume) are treated by a dedicated image processing software able to identify and interpolate the droplet profile. These measurements allow for evaluation of the resulting forces acting on the droplet due to weight, adhesion, and internal pressure. In the absence of an electric field, the experimental values of the force acting on the drop agree well with the theoretical predictions. In Fig. 7, the authors plotted the

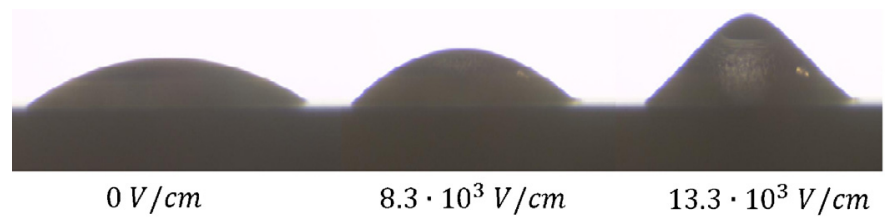

Fig. 6. Shape of an ethanol droplet for different electric field strengths [46].

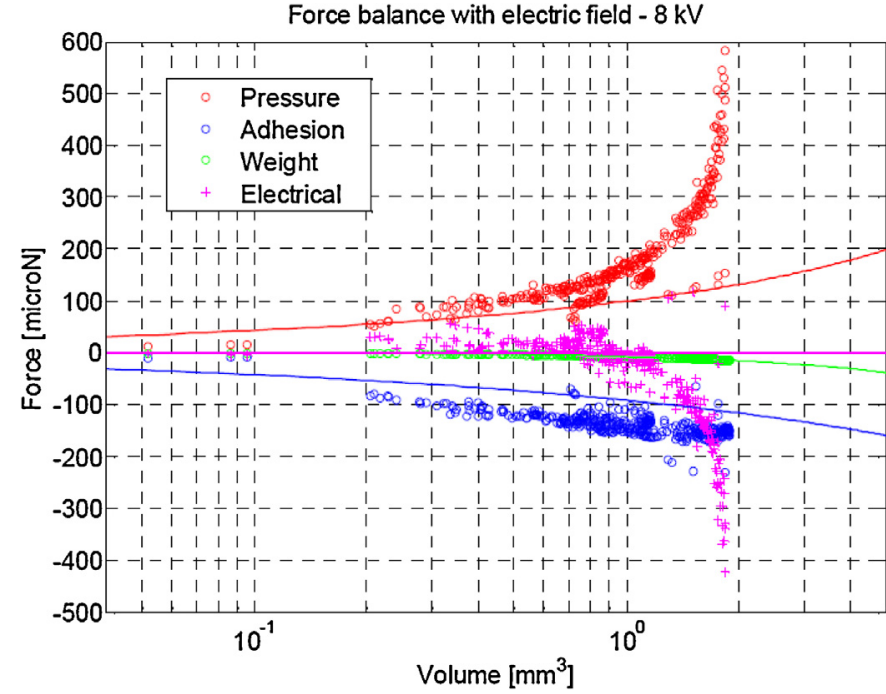

Fig. 7. Experimental values (symbols) of the resulting forces for an applied voltage of $8 \mathrm{kV}$. The theoretical values are referred to the $0 \mathrm{kV}$ case [46].

experimental values of the resulting forces on a drop subjected to an electric field of $13.3 \mathrm{kV} / \mathrm{cm}$ compared with the predictions of the theoretical model without taking into account the action of the electric field. It can be seen that the intensity of both the adhesion and internal pressure forces is increased in the presence of electric field. The resulting electric force is calculated as the algebraic sum of the others changed of sign, and is directed downwards, especially for drops of large volume, which the field seems to press against the surface [46].

Finally, the profile of the droplets subjected to an electric field of $13.3 \mathrm{kV} / \mathrm{cm}$ has been implemented in an FEM calculation, and the resulting force has been evaluated. This calculation reported a force of $-175 \mu \mathrm{N}$, compared with the value of $-165 \mu \mathrm{N}$ obtained from the experimental result, representing a $6 \%$ error between the numerical model and experiments. As shown in Fig. 8, the electric field is non-uniform in the cavity due to the shape of the electrode and the presence of the droplet, and its local strength can be only evaluated using numerical methods [46].

\subsection{Influence of an electric field on the evaporation rate of a drop}

The enhancement of the heat and mass transfer induced by the application of an electric field is a phenomenon of interest in a

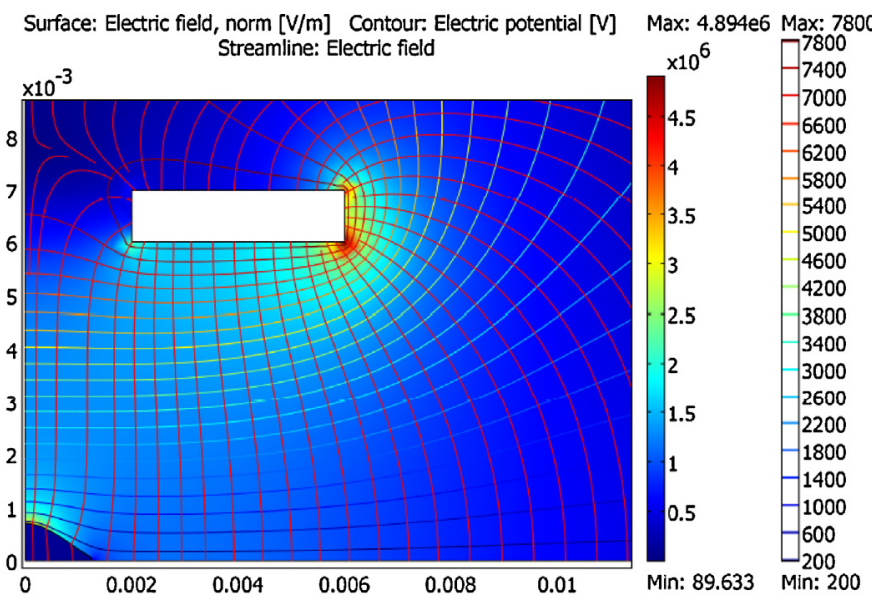

Fig. 8. Electric field distribution (COMSOL calculation) around the droplet (lower left side). The electrode (white region) is charged at $8 \mathrm{kV}$ [46]. 
wide range of industrial applications (electrospraying, cooling, heat exchange, drying, etc.). The use of an electric field in the drying process is also a promising approach to enhancing the drying rate with low energy consumption, especially in the food industry area. In this case, the electrical enhancement of the heat transfer is induced by the flow of the "corona wind", which is caused by the gas ionisation near the electrode [16]. The mean factors influencing this enhancement are the air flow velocity, the strength of the electric field, the temperature, and the nature of the evaporating fluid [16,60-63].

Although the enhancement of heat transfer by application of an electric field is well known and investigates in the electrohydrodynamic science, this intensification concerning the evaporation of sessile drops stills a subject poorly investigated and unknown. The enhancement of drop evaporation has been investigated by Takano et al. [64-67]. In these studies, liquid drops are evaporated at temperature exceeding the Leidenfrost temperature of the liquids under a voltage applied to the drop varying from $0 \mathrm{~V}$ to $2000 \mathrm{~V}$ using electrode configuration described in Fig. 1a. In their first investigation [64], the evaporation of drop of ethanol, water and cyclohexane was investigated under a applied voltage of $300 \mathrm{~V}$. The substrate temperature was $300^{\circ} \mathrm{C}$ for the evaporation of ethanol and cyclohexane drop and $400^{\circ} \mathrm{C}$ for the water drops. The highest enhancement rate corresponding to the ratio of evaporation time under no electric field to that under the electric field, was obtained with ethanol ( $\sim 20$ times), then water ( $\sim 3$ times) and cyclohexane ( $\sim 1.3$ times). In their followed study [65], their experiments was carried out using ethanol and R113 with a maximum voltage respectively of $250 \mathrm{~V}$ and $2000 \mathrm{~V}$. The maximum enhancement ratios was 7.6 for ethanol and 2.8 for R113 at the highest applied voltage. From these experiments, a quantitative interpretation of the results has not yet satisfactory been proposed. However, the authors qualitatively observed that the effect of the electric field may be less remarkable for non-polar liquids as a consequence of its high charge relaxation time. To confirm the possibility of active control of evaporation enhancement using liquids with a long relaxation time, the author studied a horizontal free surface of non-polar liquids and measured its critical voltages and response times necessary to render the surface of the liquid unstable [66]. They concluded that, even if the liquid is non-polar, surface instability can occur at a certain critical voltage and at a response time shorter than the electrical relaxation time. This finding proves the effectiveness of the electric field in enhancing and controlling the evaporation heat transfer.

\section{Conclusions and perspectives}

This review has discussed the effect of an external electric field on sessile drops. The existing studies about the influence on the contact angle, shape, and enhancement of the evaporation rate have been reviewed to plan a future investigation focused on the evaporation of sessile drops under the action of an electric field. Indeed, the enhancement of the evaporation rate by an electric field constitutes a promising and economic application involving low energetic consumption compared to the traditional evaporation process. To predict the evaporation rate, the influence of the electric field on the drop contact angle and spreading must be known. However, from the experimental results found in the literature, the effect on the contact angle has not been rigorously investigated. Nevertheless, influencing factors, such the liquid polarity, have been described. The spreading of a sessile drop subjected to an electric field has not yet been investigated. Although the enhancement of the evaporation process under the action of an electric field is well recognised, its influence on the evaporation of sessile drops has been seldom investigated.
In future investigations, the effect of the electric field on the shape and contact angle of pure liquid drops and the effect on the spreading of the drop will be determined. The effects of the experimental parameters, such as the polarity, nature of the substrate, and drop volume, will be explored; the liquid polarity could be a limiting factor in applications, the nature of the substrate can change the wettability of the liquid, and the shape of the drop resulting from the action of the electric force may be a function of its volume. The experimental results will be compared with theoretical and numerical models to predict this influence on the evaporation process.

After this preliminary phase, the evaporation phenomenon under an applied electric field will be investigated with the aim of controlling the evaporation process with good accuracy. The enhancement ratio will be calculated using a rigorous experimental protocol and by controlling the experimental parameters. Once the evaporation enhancement is understood on a fundamental level, the same experimental series will be performed without the gravity effect. Models to predict the enhancement of the evaporation rate will also be developed.

Finally, the evaporation of nano-fluids under an electric field will be studied to control (nano)particle deposition. The same research strategy will be proposed by considering the additional parameters of the particles, namely, their nature, concentration, and size.

\section{Acknowledgements}

The authors thank CNES for their financial support through the "Sessile Drop Evaporation" project, as well as ESA through the "Drop Evaporation" project.

\section{References}

[1] R.G. Picknett, R. Bexon, The evaporation of sessile or pendant drops in still air, J. Colloid Interface Science 61 (1977) 336-350.

[2] B. Sobac, D. Brutin, Triple-line behavior and wettability controlled by nanocoated substrates: influence on sessile drop evaporation, Langmuir 27 (2011) 14999-15007.

[3] S. Semenov, V.M. Starov, R.G. Rubio, H. Agogo, M.G. Velarde, Evaporation of sessile water droplets: universal behavior in presence of contact angle hysteresis, Colloids Surf. A: Physiochem. Eng. Aspects 391 (2011) 135-144.

[4] S. David, K. Sefiane, M.E.R. Shanahan, On the dynamics of wetting of water-methanol volatile sessile drops on smooth substrates, Defect Diffus. Forum 258-260, 469-473 (2006).

[5] S. David, K. Sefiane, L. Tadrist, Experimental investigation of the effect of thermal properties of the substrate in the wetting and evaporation of sessile drops, Colloids Surf. A: Physiochem. Eng. Aspects 298 (2007) 108-114.

[6] G.J. Dunna, S.K. Wilson, B.R. Duffy, S. David, K. Sefiane, A mathematical model for the evaporation of a thin sessile liquid droplet: comparison between experiment and theory, Colloids Surf. A: Physiochem. Eng. Aspects 323 (2008) 50-55.

[7] D. Brutin, B. Sobac, F. Rigollet, C. Le Nilliot, Infrared visualization of therma motion inside a sessile drop deposited onto a heated surface, Exp. Thermal Fluid Sci. 35 (2012) 521-530.

[8] B. Sobac, D. Brutin, Thermal effects of the substrate on water droplet evaporation, Phys. Rev. E 86 (2012) 021602

[9] R.D. Deegan, O. Bakajin, T.F. Dupont, G. Hubert, S.R. Nagel, T.A. Witten, Contact line deposits in an evaporating drop, Phys. Rev. E 62 (2000) 756-765.

[10] H. Hu, R.G. Larson, Evaporation of a sessile droplet on a substrate, J. Phys. Chem. B 106 (2002) 1334-1344.

[11] Y.O. Popov, Evaporative deposition patterns: spatial dimensions of the deposit, Phys. Rev. E 71 (2005) 036313.

[12] M.K. Bologa, V.V. Pushkov, A.B. Berkov, Electric field induced heat transfer enhancement in a gas-solid suspension heat exchanger, Int. J. Heat Mass Transf Vol. 28 (1985) 1245-1255.

[13] E.R.G. Eckert, Co, Heat transfer - a review of 1993 literature, Int. J. Heat Mass Transf. 39 (1996) 885-963.

[14] Y. Liu, Co, Manipulation of nanoparticles and biomolecules by electric field and surface tension, Comput. Methods Appl. Mech. Eng. 197 (2008) 2156-2172.

[15] F. Mugele, J.-C. Baret, Electrowetting: from basics to applications, J. Phys.: Condens. Matter 17 (2005) R705-R774.

[16] S.A.O. Ahmedou, O. Rouaud, Assessment of the electrohydrodynamic drying process, Food Bioprocess Technol. 2 (2009) 240-247.

[17] A. Bateni, A. Amirfazli, A.W. Neumann, Effect of an electric field on the surface tension conducting drops, Colloids Surf. A: Physiochem. Eng. Aspects 289 (2006) 25-38. 
[18] E. Borzabadi, A.G. Bailey, The profiles of axially symmetric electrified pendant drops, J. Electrostat. 5 (1978) 369-380.

[19] K. Adamiak, Interaction of two dielectric or conducting droplets aligned in the uniform electric field, J. Electrostat. 51-52 (2001) 578-584.

[20] R. Digilov, Charge-induced modification of contact: The secondary electrocapillary effect, Langmuir 16 (2000) 6719-6723.

[21] K. Hyoung, Kang How electrostatic fields change contact angle in electrowetting, Langmuir 18 (2002) 10318-10322.

[22] C. Quilliet, B. Berge, Electrowetting: a recent outbreak, Curr.Opin. Colloid Interface Sci. 6 (2001) 34-39.

[23] R. Shamai, D. Andelman, B. Berge, R. Hayes, Water, electricity, and between. . On electrowetting and its applications, Soft Matter 4 (2007) 38-45.

[24] F. Mugele, M. Duits, D. van den Ende, Electrowetting: a versatile tool for drop manipulation, generation, and characterization, Adv. Colloid Interface Sci. 161 (2010) 115-123.

[25] J. Lee, H. Moon, J. Fowler, T. Schoellhammer, C.-J. Kim, Electrowetting and electrowetting-on-dielectric for microscale liquid handling, Sens. Actuat. A 95 (2002) 259-268.

[26] P. Paik, V.K. Pamula, M.G. Pollack, R.B. Fair, Electrowetting-based droplet mixers for microfluidic systems, Lab Chip 3 (2003) 28-33.

[27] S.K. Cho, H. Moon, C.J. Kim, Creating, transporting, cutting, ans merging liquid droplets by electrowetting-based actuation for digital microfluidic circuits, J. Microelectromech. Syst. 12 (2003) 70-80.

[28] M.G. Pollack, R.B. Fair, A.D. Shenderov, Electrowetting-based actuation of liquid droplets for microfluidic applications, Appl. Phys. Lett. 77 (2000) $1725-1726$.

[29] B. Berge, J. Peseux, Variable focal lens controlled by an external voltage: an application of electrowetting, Eur. Phys. J. E. 3 (2000) 159-163.

[30] S.-L. Lee, C.-F. Yang, Numerical simulation for meniscus shape and optical performance of a MEMS-based liquid micro-lens, Opt. Express 16 (2008).

[31] T.B. Jones, An electromechanical interpretation of electrowetting, J. Micromech. Microeng. 15 (2005) 1184-1187.

[32] T.B. Jones, More about the electromechanics of electrowetting, Mech. Res. Commun. 36 (2009) 2-9.

[33] D. Chakraborty, G. Sai Sudha, S. Chakraborty, S. DasGupta, Effect of submicron particles on electrowetting on dielectrics (EWOD) of sessile droplets, J. Colloid Interface Sci. 363 (2011) 640-645.

[34] A.I. Drygiannakis, A.G. Papathanasiou, A.G. Boudouvis, Manipulation equilibrium shape transitions of microdroplets in electrowetting - a computational analysis, Microelectr. Eng. 86 (2009) 1365-1367.

[35] A. Quinn, R. Sedev, J. Ralston, Contact angle saturation in electrowetting, J. Phys, Chem. B 109 (2005) 6268-6275.

[36] H. Moon, Low voltage electrowetting-on-dielectric, J. Appl. Phys. 92 (2002) 4080-4087.

[37] R. Sedev, Electrowetting: electrocapillarity, saturation, and dynamics, Eur. Phys. J. Spec. Top. 197 (2011) 307-319.

[38] W.K. Panofsky, M. Phillips, Classical Electricity and Magnetism, AddisonWesley, Reading, 1962.

[39] D. Landau, E.M. Lifsitz, Electrodynamics of Continuous Media, Pergamon, New York, 1984.

[40] M.T. Harris, O.A. Basaran, Equilibrium shapes and stability of nonconducting pendant drops surrounded by a conducting fluid in an electric field, J. Colloid Interface Sci. 170 (1995) 308-319.

[41] O.A. Basaran, L.E. Scriven, Axisymmetric shapes and stability of pendant and sessile drops in an electric field, J. Colloid Interface Sci. 140 (1990) 10-30.

[42] H.J.J. Verheijen, M.W.J. Prins, Reversible electrowetting and trapping of charge: model and experiments, Langmuir 15 (1999) 6616-6620.

[43] W.J.J. Welters, L.G.J. Fokkink, Fast electrically switchable capillary effects, Langmuir 14 (1998) 1535-1538.
[44] V. Peykov, A. Quinn, J. Ralston, Electrowetting: a model for contact-angle saturation, Colloid Polym. Sci. 278 (2000) 789-793.

[45] A. Bateni, S. Laughton, H. Tavana, S.S. Susnar, A. Amirfazli, A.W. Neumann, Effect of electric fields on contact angle and surface tension of drops, J. Colloid Interface Sci. 283 (2005) 215-222.

[46] P. Di Marco, F. Pedretti, G. Saccone, Effect of an external electric field on the shape of a dielectric sessile drop Transfer, Fluid Mechanics, and Thermodynamics, in: 8th World Conference on Experimental Heat Transfer, June 16-20, 2013, Lisbon, Portugal, 2013.

[47] J.M. Roux, J.L. Achard, Y. Fouillet, Forces and charges on an undeformable droplet in the DC field of a plate condenser, J. Electrostat. 66 (2008) 283-293.

[48] J.M. Roux, J.L. Achard, Forces and charges on a slighty deformed droplet in the DC field of a plate condenser, J. Electrostat. 67 (2009) 789-798.

[49] A. Gliere, J.M. Roux, J.L. Achard, Lift-off of a Conducting Sessile Drop in An Electric Field Microfluid Nanofluid, 2013, http://dx.doi.org/10.1007/ s10404-013-1144-6, February, Published online.

[50] C. Roero, Contact angle measurements of sessile drops deformed by a DC electric field, Cont. Angle, Wettab. Adhes. 4 (2006) 165-176.

[51] A. Bateni, S.S. Susnar, A. Amirfazli, A.W. Neumann, Development of a new methodology to study drop shape and surface tension in electric fields, Langmuir 20 (2004) 7589-7597.

[52] A. Bateni, A. Ababneh, J.A.W. Elliott, A.W. Neumann, A. Amirfazli, Effect of gravity and electric field $n$ shape and surface tension of drops, Adv. Space Res. 36 (2005) 64-69.

[53] L. Rayleigh, On the equilibrium of liquid conducting masses charged with electricity, Phil. Mag. 14 (1882) 184-195.

[54] J.R. Melcher, G.I. Taylor, Electrohydrodynamics: a review of the role of interfacial shear stresses, Ann. Rev. Fluid Mech. 1 (1969) 111.

[55] C.E. Rosenkilde, A dielectric fluid drop in an electric field, Proc. R. Soc. Lond. A 3112 (1969) 473.

[56] M.J. Miksis, Shape of a drop in an electric field, Phys. Fluids 24 (1981) 1967.

[57] F.K. Wohlhuter, O.A. Basaran, Shapes and stability of pendant and sessile dielectric drops in an electric field, J. Fluid Mech. 235 (1992) 481-510.

[58] O.A. Basaran, F.K. Wohlhuter, Effect of nonlinear polarization on shapes and stability of pendant and sessile drops in an electric (magnetic) field, J. Fluid Mech. 244 (1992) 1-16.

[59] S.N. Reznik, A.L. Yarin, A. Theron, E. Zussman, Transient and steady shapes of droplets attached to a surface in a strong electric field, J. Fuid Mech. 516 (2004) 349-377.

[60] N.N. Barthakur, S. Bhartendu, Enhancement of evaporation rates from thin layers of liquids exposed to air ions, Int. J. Biometeorol. 32 (1988) 163-167.

[61] W. Cao, Y. Nishyama, S. Koide, Electrohydrodynamic drying characteristics of wheat using high voltage electrostatic field, J. Food Eng. 62 (2004) 209-213.

[62] W. Cao, Y. Nishyama, S. Koide, Z.H. Lu, Drying enhancement of rough rice by electric field, Biosyst. Eng. 87 (2004) 445-451.

[63] T.I.J. Goodenough, P.W. Goodenough, S.M. Goodenough, The efficiency of corona drying and its application to the food industry, J. Food Eng. 80 (2007) 1233-1238.

[64] K. Takano, I. Tanasawa, S. Nishio, Enhancement of a droplet from a hot surface by electric field, Proceedings of the Ninth International Heat Transfer Conference, vol. 4, 69-74,90.

[65] K. Takano, I. Tanasawa, S. Nishio, Active enhancement of evaporation of a liquid drop on a hot solid surface using a static electric field, Int. J. Heat Mass Trans. 37 (Suppl. 1) (1994) 65-71.

[66] K. Takano, I. Tanasawa, Enhancement of evaporation of a droplet using EHD effect, JSME Int. J. B 38 (1995) 288-294.

[67] K. Takano, I. Tanasawa, S. Nishio, Enhancement of evaporation of a liquid droplet using EHD effect: criteria for instability of gas-liquid interface under electric field, J. Enhanc. Heat Transf. 3 (1996) 73-81. 